\title{
Estrategias de afrontamiento en adolescentes gallegos
}

\author{
Antonio López Castedo, Ma Esther López Pérez \\ Facultad de Ciencias de la Educación (Campus de Ourense). Universidad de Vigo
}

\begin{abstract}
Resumen
El presente trabajo analiza la incidencia que sexo y centro educativo tienen sobre las estrategias de afrontamiento en 447 adolescentes entre 12 y 16 años a los que se les aplicó el Test de Evaluación de Afrontamiento en Adolescentes de Frydenberg y Lewis (1993). Los resultados encontrados sugieren que las mujeres manejan más estrategias que los hombres. A su vez, en el centro educativo se observa que la mayoría de las estrategias son empleadas por adolescentes de centros privados. Se aprecian diferencias significativas en ambas variables. En la discusión se analizan las implicaciones a la luz de los resultados hallados.

Palabras clave: estrategias de afrontamiento, adolescencia, educación.
\end{abstract}

\section{Introducción}

El estudio de las estrategias de afrontamiento, entendidas como habilidades para afrontar y resolver un problema, regular las emociones o manejar dificultades para reducir sus efectos cuenta con modelos e instrumentos elaborados específicamente para adolescentes en los que se tienen en cuenta las peculiaridades de los cambios que se producen en esta etapa evolutiva, los aspectos que determinan que esos cambios sean percibidos como estresantes o no, así como las características y la relevancia de recursos personales y sociales disponibles para hacer frente a los estresores (Compas, Connor-Smith, Saltzman, Thomsen y Wadsworth, 2001; Cuadra-Peralta, Ochoa de la Maza, Cornejo y Barrera, 2009; Frydenberg, 1997; Gónzalez, Montoya, Casullo y Bernabéu, 2002). Esta función moderadora desempeña un papel importante mediador entre las experiencias estresantes a las que están sometidas las personas, los recursos personales y sociales con los que cuentan para hacerles frente y las consecuencias que se derivan para la salud física y psicológica de las personas (Sandín, 2003).

En el campo educacional se presta cada vez mayor interés al estudio de las estrategias de afrontamiento debido a la relevancia que adquieren en los adolescentes al tener que enfrentarse a los desafíos en el curso del proceso de maduración, que involucra cambios físicos, psicológicos, cognoscitivos y sociales, sobre todo si se contemplan las presiones y estrés creciente al que están sometidos en ambientes de estudio y trabajo cada vez más competitivos (Aunola, Stattin y Nurmi, 2000; Frydenberg y Lewis, 1996, 1999; Plancherel y Bolognini, 1995; Richaud de Minzi, 2004).

Desde esta perspectiva, se hace necesario conocer y profundizar cómo el sexo y el tipo de centro pueden influir en las estrategias de afrontamiento del alumnado de secundaria.

\section{Participantes \\ Método}

La población objeto de estudio se corresponde con los alumnos/as de Educación Secundaria Obligatoria (ESO) de las provincias de Ourense y Lugo, con edades comprendidas entre los 12 y 16 años. Se seleccionó una muestra de un total de 447 alumnos/as, de centros públicos y privados-concertados y que voluntariamente accedieron a colaborar en este estudio. La caracterización de la muestra se distribuye de la siguiente forma: según el sexo se observa una distribución bastante equilibrada, con un ligero predominio de las mujeres que representan el 52.1\% (233) y los hombres el 47.9\% (214) del total. En cuanto a la edad, entre 13 y 15 años se aglutinan el 68.1\% de la muestra, siendo los grupos más reducidos los de 16 y 12 años. La edad media es de 13.83 años, con una desviación típica de 1.38. Con relación al tipo de centro en el que estudian, se observa un porcentaje superior en los centros privados-concertados (67.3\%) frente a los públicos (32.7\%). Respecto al curso, se constata que el grupo más numeroso es el formado por $2^{\circ}$ de la ESO con un $30 \%$ (134), seguidos por $1^{\circ}$ con un $27.5 \%$, mientras que $3^{\circ}$ y $4^{\circ}$ su distribución es bastante homogénea, con un $21.7 \%$ y $20.8 \%$, respectivamente.

\section{Instrumentos}

En primer lugar, se utilizó un Cuestionario estructurado "ad hoc" relativo a las variables sociodemográficas entre las que se incluyen sexo, edad, tipo de centro y curso. En segundo lugar, la versión castellana abreviada de la Adolescent Coping Scale (ACS) de Frydenberg y Lewis (1993) y realizada por Richaud de Minzi (2003). Está compuesta por 46 ítems con formas diferentes con las que la gente suele encarar sus problemas o preocupaciones. Se utiliza una escala Likert con 5 alternativas, incluyendo una sexta opción de irrelevancia con el problema.

Los ítems correspondientes a estos factores fueron analizados desde los modelos de Billings y Moos, que parten de la división del afrontamiento en: Focalizado en la evaluación, Focalizado en el problema y Focalizado en la emoción a la que agregan. Dentro de estas tres grandes categorías definen nueve subdimensiones: Análisis lógico, Reevaluación cognitiva y Evasión cognitiva (Focalizado en la evaluación); Requerimiento de información o asesoramiento, Acción y Desarrollo de gratificaciones 
alternativas (Focalizado en el problema), Regulación afectiva, Aceptación con resignación y Descarga emocional (Focalizado en la emoción). Una vez determinadas las nuevas escalas, se analizó la consistencia interna de cada una, obteniéndose alfas de Cronbach que oscilaron entre .56 y .70 (Richaud de Minzi, 2003).

Está formada por 11 estrategias que se refieren a:

\begin{tabular}{|c|c|c|}
\hline Estrategias & Interpretación & Indicador \\
\hline $\begin{array}{l}\text { Reestructuració } \\
\text { n cognitiva }\end{array}$ & $\begin{array}{c}\text { Buscar los aspectos } \\
\text { positivos de las } \\
\text { situaciones, viendo el } \\
\text { lado bueno de las cosas } \\
\text { y considerarse } \\
\text { afortunado. }\end{array}$ & $\begin{array}{l}\text { Ítems: } 20 \text {, } \\
24,32,44 .\end{array}$ \\
\hline Autoinculparse & $\begin{array}{c}\text { Verse como responsable } \\
\text { de los problemas o } \\
\text { preocupaciones que } \\
\text { tienen. }\end{array}$ & $\begin{array}{l}\text { Ítems: 7, 29, } \\
37 .\end{array}$ \\
\hline Fatalismo & $\begin{array}{l}\text { Buscar apoyo espiritual } \\
\text { a través de la oración y } \\
\text { la creencia, la ayuda de } \\
\text { un líder o de Dios. }\end{array}$ & $\begin{array}{l}\text { Ítems: 9, 15, } \\
31,35 .\end{array}$ \\
\hline $\begin{array}{l}\text { Evasión a } \\
\text { través de la } \\
\text { diversión }\end{array}$ & $\begin{array}{c}\text { Invertir en amigos y } \\
\text { comenzar relaciones } \\
\text { estables. }\end{array}$ & $\begin{array}{l}\text { Ítems: 3, 22, } \\
\text { 26, } 43 .\end{array}$ \\
\hline $\begin{array}{l}\text { Focalización } \\
\text { del } \\
\text { problema }\end{array}$ & $\begin{array}{l}\text { Buscar apoyo social } \\
\text { compartiendo el } \\
\text { problema, } \\
\text { concentrándose en } \\
\text { resolverlo }\end{array}$ & $\begin{array}{l}\text { Ítems: } 1,10 \text {, } \\
\text { 12, 13, 19, } \\
40,41 .\end{array}$ \\
\hline $\begin{array}{l}\text { Evasión a } \\
\text { través de la } \\
\text { actividad } \\
\text { Física }\end{array}$ & $\begin{array}{c}\text { Realización de la } \\
\text { práctica deportiva para } \\
\text { mantenerse en forma. }\end{array}$ & $\begin{array}{l}\text { Ítems: 11, } \\
21,23,33 .\end{array}$ \\
\hline $\begin{array}{l}\text { Búsqueda } \\
\text { de apoyo } \\
\text { emocional }\end{array}$ & $\begin{array}{l}\text { Preocupación e interés } \\
\text { por su relaciones con } \\
\text { los demás, en general, y } \\
\text { por lo que los otros } \\
\text { piensan }\end{array}$ & $\begin{array}{l}\text { Ítems: } 4,14 \text {, } \\
27,35 .\end{array}$ \\
\hline $\begin{array}{l}\text { Descarga } \\
\text { emocional y } \\
\text { somatización }\end{array}$ & $\begin{array}{l}\text { Intento de sentirse } \\
\text { mejor y reducir la } \\
\text { tensión. }\end{array}$ & $\begin{array}{l}\text { Ítems: 5, 36, } \\
45,46 .\end{array}$ \\
\hline Ansiedad & $\begin{array}{l}\text { Caracterizada por lo } \\
\text { elementos que indican } \\
\text { preocupación por el } \\
\text { futuro en términos } \\
\text { generales. }\end{array}$ & $\begin{array}{l}\text { Ítems: 2, 34, } \\
42 .\end{array}$ \\
\hline Aislamiento & $\begin{array}{l}\text { Huida de los demás y el } \\
\text { deseo de que no } \\
\text { conozcan sus } \\
\text { problemas. }\end{array}$ & $\begin{array}{l}\text { Ítems: } 8,18 \text {, } \\
30,38 .\end{array}$ \\
\hline No acción & $\begin{array}{l}\text { Rechazo consciente de } \\
\text { la existencia de un } \\
\text { problema. }\end{array}$ & $\begin{array}{l}\text { Ítems: } 6,16 \text {, } \\
17,28 .\end{array}$ \\
\hline
\end{tabular}

\section{Procedimiento}

Previamente a la recogida de dato, se procedió a contactar con el equipo directivo y los orientadores de los centros, con el objeto de explicarles la finalidad y alcance de la investigación y proponerles su participación voluntaria. Obtenido el consentimiento de la dirección de los centros, se mantiene una reunión informativa para dar a conocer el estudio y que éstos lo trasladen a toda la comunidad educativa. Los tutores participan de forma voluntaria y no remunerada. Paralelamente, se informa a los padres sobre la investigación y se obtiene el consentimiento de la participación de sus hijos en el estudio. A los adolescentes que deciden colaborar se les pide que sean lo más sinceros posible a la hora de contestar y que no dejen ninguna pregunta sin responder. Las instrucciones explicativas son las mismas en todas las aulas y el mismo encuestador, a fin de evitar un factor de sesgo. Es necesario señalar la receptividad de todos los profesores, alumnos y padres a los que se les solicitó colaboración, así como la alta participación de los estudiantes en esta investigación.

\section{Diseño y análisis de datos}

El diseño empleado en esta investigación es no experimental, es decir, se trata de observar los las estrategias de afrontamiento de los adolescentes en su ambiente natural (centros educativos) para después analizarlos y, además, de enfoque cuantitativo, usando la recogida de datos para probar hipótesis, con base a una medición numérica y el análisis estadístico, que ayuden a establecer patrones de comportamiento. A su vez, es de tipo transversal, descriptivo y analítico.

Para analizar los datos obtenidos, se sometieron los resultados a los siguientes análisis estadísticos: análisis de frecuencias y porcentajes de cada variable cualitativa, puntuación media y desviación típica de cada variable cuantitativa, prueba "t" de muestras independientes. Para todos los análisis que se considero que una relación era estadísticamente significativa cuando $p<.05$ y no significativa si $p>.05$. L os datos fueron analizados mediante el programa estadístico SPSS 21.9 para Windows.

\section{Resultados}

Por lo que respecta a las diferentes estrategias de afrontamiento en Adolescentes (ACS) en función del sexo (tabla 1), se pone de manifiesto que en Reestructuración cognitiva, Fatalismo, Evasión a través de la diversión, Focalización del problema, Búsqueda de apoyo emocional, Descarga emocional y somatización, y Ansiedad la media más alta corresponde a las mujeres en relación a los hombres. Por otra parte, en Autoinculparse, Evasión a través de la actividad física, Aislamiento y No acción, se observa que la puntuación mayor corresponde a los hombres en relación a las mujeres. 
Tabla 1

Medias, desviaciones típicas y prueba " $t$ " de las estrategias de la ACS en función del Sexo

\begin{tabular}{lccc}
\hline \multicolumn{1}{c}{ Estrtategias } & Sexo & $\bar{X}(S x)$ & $\mathrm{t}(p)$ \\
\hline Reestructuración & Mujer & $14.76(3.66)$ & 1.386 \\
cognitiva & Hombre & $14.34(3.94)$ & $(.240)$ \\
Autoinculparse & Mujer & $6.93(3.28)$ & .101 \\
& Hombre & $6.96(3.20)$ & $(.831)$ \\
Fatalismo & Mujer & $9.87(3.20)$ & 1.119 \\
Evasión a través & Hombre & $9.54(3.38)$ & $(.291)$ \\
de la diversión & Mujer & $14.19(3.83)$ & .664 \\
Focalización del & Mujer & $13.90(3.84)$ & $(.416)$ \\
problema & Hombre & $24.44(5.80)$ & 4.916 \\
Evasión a través & Mujer & $12.80(4.30)$ & $(<.027)$ \\
de la actividad & Hombre & $13.40(4.22)$ & 2.262 \\
física & & & $(.133)$ \\
Búsqueda de & Mujer & $15.96(3.83)$ & 6.383 \\
apoyo emocional & Hombre & $15.03(3.98)$ & $(<.012)$ \\
Descarga & Mujer & $9.33(3.76)$ & 13.105 \\
emocional y & Hombre & $8.06(3.65)$ & $(<.000)$ \\
somatización & & & \\
Ansiedad & Mujer & $11.00(2.96)$ & 4.919 \\
& Hombre & $10.36(3.10)$ & $(<.027)$ \\
Aislamiento & Mujer & $9.36(3.57)$ & 2.563 \\
No acción & Hombre & $9.94(4.01)$ & $(.110)$ \\
& Mujer & $6.74(3.08)$ & 11.780 \\
& Hombre & $7.81(3.51)$ & $(<.001)$ \\
\hline
\end{tabular}

La prueba "t" de muestras independientes, nos indica que existen diferencias significativas en Focalización del problema ( $\mathrm{t}=4.916 ; p<.027)$, Búsqueda de apoyo emocional $(\mathrm{t}=6.383 ; p<.012)$, Descarga emocional $\mathrm{y}$ somatización ( $\mathrm{t}=13.105 ; p<.000)$, Ansiedad $(\mathrm{t}=4.919$; $p<.027)$, y No acción ( $\mathrm{t}=11.780 ; p<.001)$. Dado que los resultados de la prueba " $\mathrm{t}$ " muestran diferencias significativas $(p<.05)$ en Focalización del problema, Búsqueda de apoyo emocional, Descarga emocional y somatización y Ansiedad, éstas se ponen de manifiesto con una media más alta de las mujeres en relación a los hombres; por el contrario, en la estrategia de No acción, hombres presentan una puntuación más alta que las mujeres.

En cuanto a las distintas estrategias de la ACS en función del Tipo de Centro (tabla 2), se puede observar que todas las estrategias (Reestructuración cognitiva, Autoinculparse, Fatalismo, Evasión a través de la diversión, Focalización del problema, Evasión a través de la actividad física, Búsqueda de apoyo emocional, Descarga emocional y somatización, Ansiedad) son utilizadas por los adolescentes de centros privadosconcertados, a excepción de Aislamiento y No acción.

La prueba " $\mathrm{t}$ " de muestras independientes, nos indica que se presentan diferencias significativas en Reestructuración cognitiva $(\mathrm{t}=6.077 ; p<.014)$, Autoinculparse $\quad(\mathrm{t}=3.842 ; p<.050)$, Fatalismo $(\mathrm{t}=$ 7.127; $p<.008)$, Focalización del problema $(\mathrm{t}=19.436$; $p<.000$ ), Evasión a través de la actividad física ( $\mathrm{t}=$ 9.678; $p<.002)$, Búsqueda de apoyo emocional $(\mathrm{t}=$ 12.173; $p<.001)$, Ansiedad ( $\mathrm{t}=15.174 ; p<.000)$, y No acción $(\mathrm{t}=4.577 ; p$-033). Dado que los resultados de la prueba "t" exhiben diferencias significativas ( $p$.05) en Reestructuración cognitiva, Autoinculparse, Fatalismo, Focalización del problema, Evasión a través de la actividad física, Búsqueda de apoyo emocional, Ansiedad y No acción, éstas son empleadas por los adolescentes de centros privados-concertados con una media más alta en relación a los públicos.

Tabla 2

Medias, desviaciones típicas y prueba " $t$ " de las estrategias de la ACS atendiendo al Tipo de Centro

\begin{tabular}{|c|c|c|c|}
\hline Estrategias & Centro & $\overline{\bar{X}}(S x)$ & $\mathrm{t}(p)$ \\
\hline \multirow{2}{*}{$\begin{array}{l}\text { Reestructuración } \\
\text { cognitiva }\end{array}$} & Público & 13.92 (3.84) & 6.077 \\
\hline & Privado-C & 14.86 (3.75) & $(<.014)$ \\
\hline \multirow{2}{*}{ Autoinculparse } & Público & 6.51 (3.34) & 3.842 \\
\hline & Privado-C & 7.15 (3.18) & $(<.050)$ \\
\hline \multirow{2}{*}{ Fatalismo } & Público & 9.12 (3.53) & 7.127 \\
\hline & Privado-C & 9.99 (3.13) & $(<.008)$ \\
\hline \multirow{4}{*}{$\begin{array}{l}\text { Evasión a través } \\
\text { de la diversión } \\
\text { Focalización del } \\
\text { problema }\end{array}$} & Público & 13.66 (4.03) & 2.214 \\
\hline & Privado-C & 14.22 (3.73) & $(.137)$ \\
\hline & Público & 22.05 (6.61) & 19.436 \\
\hline & Privado-C & 24.69 (5.61) & $(<.000)$ \\
\hline \multirow{2}{*}{$\begin{array}{l}\text { Evasión a través } \\
\text { de la actividad } \\
\text { física }\end{array}$} & Público & 12.21 (4.32) & \\
\hline & Privado-C & 13.51 (4.05) & $(<.002)$ \\
\hline \multirow{2}{*}{$\begin{array}{l}\text { Búsqueda de } \\
\text { apoyo } \\
\text { emocional }\end{array}$} & Público & $14.60(4.00)$ & \\
\hline & Privado-C & 15.96 (3.81) & $(<.001)$ \\
\hline \multirow{2}{*}{$\begin{array}{l}\text { Descarga } \\
\text { emocional y } \\
\text { somatización }\end{array}$} & Público & 8.27 (3.75) & \\
\hline & Privado-C & 8.94 (3.74) & $\begin{array}{l}5.144 \\
(.077\end{array}$ \\
\hline \multirow[t]{2}{*}{ Ansiedad } & Público & $9.90(3.15)$ & 15.174 \\
\hline & Privado-C & 11.08 (2.91) & $(<.000)$ \\
\hline \multirow{2}{*}{ Aislamiento } & Público & 9.84 (3.94) & .617 \\
\hline & Privado-C & 9.54 (3.73) & (.433) \\
\hline \multirow[t]{2}{*}{ No acción } & Público & 7.73 (3.72) & 4.577 \\
\hline & Privado-C & $7.02(3.11)$ & $(<.033)$ \\
\hline
\end{tabular}

\section{Discusión}

Este trabajo ha centrado su interés en identificar las estrategias de afrontamiento que utilizaron los adolescentes.

En relación con la consistencia interna de la escala total evaluada mediante el alfa de Cronbach, se encontró un índice de .85 y para las distintas dimensiones entre .62 y .75, en la misma línea a los hallados por Frydenberg y Lewis (1997), Casullo y Fernández (2000), Richaud de Minzi (2003) y Guerreiro, Cruz, Figueira y Sampaio (2014). Por tanto, consideramos que este estudio alberga unos índices de fiabilidad muy satisfactorios.

En cuanto al sexo, los datos encontrados varían de unos estudios a otros, aunque presentan cierto grado de coincidencia. Las mujeres utilizan por término medio más estrategias de afrontamiento (Moysén, Garay, Esteban y Gurrula, 2011), teniendo predilección por la Reestructuración cognitiva, Fatalismo, Evasión a través de la diversión, Focalización del problema, Búsqueda de apoyo emocional, Descarga emocional y somatización, y Ansiedad. Por el contrario, los hombres emplean un afrontamiento centrado en Autoinculparse, Evasión a través de la actividad física, Aislamiento y No acción. Estos hallazgos son congruentes con la propuesta de 
Frydenberg (1997) según la cual, al inicio de la adolescencia, los importantes cambios que se producen en el ámbito académico y social son percibidos como retos y oportunidades $\mathrm{y}$, en general, se afrontan con optimismo y esperanza; sin embargo, a medida que se avanza en la adolescencia, muchas de las expectativas iniciales se ven frustradas y el optimismo inicial se ve sustituido por una visión más pesimista. La Psicología evolutiva hace hincapié que en este período es importante el establecimiento de la propia identidad. Por ello, en las primeras etapas de la adolescencia tiene mucha relevancia la identificación con el grupo de iguales, lo que incrementa la susceptibilidad a la influencia del grupo que, posteriormente, se va reduciendo paulatinamente (Coleman y Hendry, 1999).

Por otra parte, los resultados obtenidos al comparar las estrategias de afrontamiento entre mujeres y hombres ratifican otros estudios al utilizar este instrumento (Frydenberg y Lewis, 1993, 1996; González et al., 2002) y apuntan a que la tendencia en las mujeres de apoyarse en otros para encarar sus problemas (Plancherel, Bolognini y Halfon, 1998; Frydenberg y Lewis, 1999; Recklitis y Noam, 1999; Washburn, 2000) y la de los varones a despreocuparse e intentar resolver las situaciones por sí mismos ya está presente al inicio de la adolescencia (Frydenberg y Lewis, 1999; Navarro, 2001; Recklitis y Noam, 1999),

El contexto educativo en que se desenvuelve el adolescente pudiera tener un impacto importante en la elección de las estrategias de afrontamiento utilizadas. De esta manera, según el tipo de centro, se encontró que todas las estrategias, a excepción de Aislamiento y No acción, son utilizadas por los adolescentes de centros privados-concertados. Veloso-Besio, Caqueo-Arancibia, Caqueo-Urízar, Muñoz-Sánchez y Villegas-Abarzúa (2010), obtuvieron resultados similares, lo que podría estar relacionado con el contexto psicosocial en el que se encuentran inmersos los jóvenes (Cohen y Figueroa, 2004) o al nivel socio económico al que pertenecen (Richaud de Minzi, 2005).

Los hallazgos encontrados en el estudio son sugerentes y resultan orientadores a la hora de diseñar programas de prevención al inicio de la adolescencia. Desde un enfoque preventivo, la identificación de las estrategias de afrontamiento con que cuenta el adolescente, nos llevan a considerar los recursos de que disponen éstos y que funcionan como factores protectores de su salud (Seligman y Csikszentmihalyi, 2000). Los mismos contribuyen al crecimiento y desarrollo saludable, en la medida que protegen al individuo y le permiten afrontar las situaciones de riesgo que se le presenten (Sheldon y King, 2001; Snyder y Lopez, 2002). La utilización de estas estrategias no es ajena a lo que acontece en el ámbito escolar, ya que se relaciona con cuestiones tales como el análisis y la organización de la información, la comunicación de ideas, la planificación, el trabajo en equipo y la resolución de problemas o cómo buscar el consejo de los demás o hablar de los propios problemas con otros. Por tanto, es importante que se haga consciente en los educadores de la importancia de su mediación para el desarrollo de la capacidad para el afrontamiento, entendida como estrategia cognitivoconductual, con miras al logro de una adaptación y transición efectiva hacia la adultez.

\section{Referencias}

Aunola, K., Stattin, K., y Nurmi, J.E. (2000). Parenting styles and adolescents' achievement strategies. Journal of Adolescence, 23, 205-222.

Cohen, S., y Figueroa, M. I. (2004). Estrategias y estilos de afrontamiento del estrés en adolescentes. Anales de Psicología, 2, 29-52.

Coleman, J., y Hendry, L. B. (1999). The nature of adolescence ( $3^{\mathrm{a}}$ ed.). Londres: Routledge.

Compas, B. E., Connor-Smith, J. K., Saltzman, H., Thomsen, A.H., y Wadsworth, M. E. (2001). Coping with stress during childhood and adolescence: Problems, progress, and potential in theory and research. Psychological Bulletin, 127, 87-127.

Cuadra-Peralta, A., Ochoa de la Maza, O., Cornejo, M., y Barrera, R. (2009). Test de Rorscharch: Respuestas diferenciales entre pacientes con y sin intento suicida. Fractal: Revista de Psicología, 21, 457 - 486.

Frydenberg, E. (1997). Adolescent coping: Theoretical and research perspectives. Londres: Routledge.

Frydenberg, E., y Lewis, R. (1993). Boys play sport and girls turn to others: age, gender and ethnicity as determinants of coping. Journal of Adolescence, 16, 253-266.

Frydenberg, E., y Lewis, R. (1996). A replication study of the structure of the Adolescent Coping Scale: Multiple forms and applications of a self-reported inventory in a counselling and research context. European Journal of Psychological Assessment, 12, 224-235.

Frydenberg, E., y Lewis, R. (1997). Manual: Escalas de afrontamiento para adolescentes (ACS). Madrid: TEA Ediciones.

Frydenberg, E., y Lewis, R. (1999). Things don't better just because you're older: Acase for facilitating reflection. British Journal of Educational Psychology, 69, 81-94

González, R., Montoya, I., Casullo, M.M., y Bernabéu, J. (2002). Relación entre estilos y estrategias de afrontamiento y bienestar psicológico en adolescentes. Psicothema, 14, 363- 368.

Guerreiro, D.F.; Cruz, D.; Figueira, M.L., y Sampaio, D. (2014). Estudo de Adaptação e Características Psicométricas da Versão Portuguesa da Adolescent Coping Scale - Escala de Coping para Adolescentes. Acta Médica Portuguesa, 27, 166-180.

Moysén, A., Garay, J., Esteban, J.M., y Gurrula, M. (2011). Estrategias de afrontamiento en universitarios consumidores y no consumidores de alcohol. Interpsiques, 12 Congreso Virtual de Psiquiatría, Ferbrero-Marzo.

Navarro, F. (2001). Incidencia del género de los adolescentres en los conflictos, estrategias de afrontamiento, satisfacción y desadaptación escolar que experimentan. Información psicológica, 75, 4347. 
Plancherel, B., Bolognini, M., y Halfon, O. (1998). Coping strategies in early and mid-adolescence: Differences according to age and gender in a community sample. European Psychologist, 3, 192201.

Plancherel, B., y Bolognini, M. (1995). Coping and mental health in early adolescence. Journal of Adolescence, 18, 459-474.

Recklitis, C.J., y Noam, G.G. (1999). Clinical and developmental perspectives on adolescent coping. Child Psychiatry and Human Development, 30, 87101.

Richaud de Minzi, M. C. (2003). Coping Assessment in adolescents. Adolescence, 38, $321-330$.

Richaud de Minzi, M. C. (2004). Development of coping resources in childhood and adolescents. Interdisciplinaria, Número Especial, 63-74.

Richaud de Minzi, M. C. (2005). Estilos parentales y estrategias de afrontamiento en niños. Revista Latinoamericana de Psicología, 37, 47-58.

Sandín, B. (2003). El estrés: un análisis basado en el papel de los factores sociales. International Journal of Clinical and Health Psychology, 3, 141-157.
Seligman, M.E. y Csikszentmihalyi, M. (2000). Positive psychology. An introduction. American Psychologist, 55, 5-14.

Sheldon, K.M., y King, L. (2001). Why positive psychology is necessary. American Psychologist, 56, 216-217.

Snyder, C.R., y Lopez, S.J. (2002), Handbook of positive psychology. Londres: Oxford University Press.

Veloso-Besio, C., Caqueo-Arancibia, W., CaqueoUrízar, A., Muñoz-Sánchez, Z., y Villegas-Abarzúa, F. (2010). Estrategias de afrontamiento en adolescentes. Fractal: Revista de Psicología, 22, 23 34.

Washburn, J.M. (2000). The influence of gender, sexrole orientation, and self-esteem on adolescents. use of coping strategies. Dissertation Abstracts International Section A. Humanities and Social Sciences, 61(1-A), 88. 